\title{
A Motion-Compensated Orthogonal Transform with Energy-Concentration Constraint
}

\author{
Markus Flierl and Bernd Girod \\ Max Planck Center for Visual Computing and Communication \\ Stanford University, California \\ \{mflierl, bgirod\}@stanford.edu
}

\begin{abstract}
This paper discusses a transform for successive pictures of an image sequence which strictly maintains orthogonality while permitting motion compensation between pairs of pictures. The well known motion-compensated lifted Haar wavelet maintains orthogonality only approximately. In the case of zero motion fields, the motion-compensated lifted Haar wavelet is known to be orthogonal. But for complex motion fields with many multi-connected and unconnected pixels, the motioncompensated lifted Haar wavelet cannot accurately maintain its transform property and, hence, suffers a performance degradation. The presented motion-compensated orthogonal transform strictly maintains orthogonality for any motion field. Further, the transform is designed with an energy-concentration constraint. The energy of the input pictures is accumulated in the temporal low-band while the temporal high-band is zero if the input pictures are identical up to a known single-connecting motion field. This constraint makes the transform suitable for coding applications.
\end{abstract}

\section{INTRODUCTION}

We address the problem of representing image sequences for coding and communication applications. Well known methods are standard hybrid video coding techniques as well as subband coding schemes. The latter are deemed to provide more flexible representations which may better adapt to heterogeneous communication scenarios. For 3-D subband coding with motion compensation, [1] proposes to distinguish between connected, covered, and uncovered pixels when incorporating motion compensation for filtering in temporal direction. Motion-compensated filtering in [2] addresses the problem of double-connected pixels and proposes an ad-hoc method to resolve the ambiguity. [3], [4], [5] choose a lifting implementation for the temporal filter and incorporate motion compensation into the lifting steps. In contrast to previous work, the lifting implementation permits a reversible filter structure, but still, it struggles with unconnected, connected, and multi-connected pixels when performing the update step. [6] proposes an optimum update step that minimizes the mean-squared reconstruction error. But note that the motioncompensated lifted Haar wavelet maintains orthogonality only approximately. For a zero motion field, the motion-compensated lifted Haar wavelet is known to be orthogonal. But for complex motion fields with many multi-connected and unconnected pixels, the motion-compensated lifted Haar wavelet

This work has been supported by the Max Planck Center for Visual Computing and Communication. cannot accurately maintain its transform property and, hence, suffers a performance degradation.

In contrast to previous work, the presented motion-compensated orthogonal transform strictly maintains orthogonality for any motion field. The transform is factored into a sequence of incremental transforms that are strictly orthogonal. The incremental transforms maintain scale counters to keep track of the scale factors that are introduced to ensure orthogonality. The decorrelation factor of each incremental transform is determined by the scale counters and is chosen such that the transform meets an energy-concentration constraint.

The paper is organized as follows: Section II introduces the motion-compensated orthogonal transform and discusses the incremental transform as well as the energy-concentration constraint. Section III proposes an adaptive spatial transform to process the resulting temporal low-band. Section IV presents the experimental results.

\section{Motion-Compensated Orthogonal Transform}

This section discusses how the transform is factored into incremental transforms. We outline the construction of the incremental transform and the incorporation of the energyconcentration constraint.

Let $\mathbf{x}_{1}$ and $\mathbf{x}_{2}$ be two vectors representing consecutive pictures of an image sequence. The transform $T$ maps these vectors according to

$$
\left(\begin{array}{l}
\mathbf{y}_{1} \\
\mathbf{y}_{2}
\end{array}\right)=T\left(\begin{array}{l}
\mathbf{x}_{1} \\
\mathbf{x}_{2}
\end{array}\right)
$$

into two vectors $\mathbf{y}_{1}$ and $\mathbf{y}_{2}$ which represent the temporal lowand high-band, respectively. Now, we factor the transform $T$ into a sequence of $k$ incremental transforms $T_{\kappa}$ such that

$$
T=T_{k} T_{k-1} \cdots T_{\kappa} \cdots T_{2} T_{1}
$$

where each incremental transform $T_{\kappa}$ is orthogonal by itself, i.e., $T_{\kappa}^{T} T_{\kappa}=T_{\kappa} T_{\kappa}^{T}=I$ holds for all $\kappa=1,2, \cdots, k$. This guarantees that the transform $T$ is also orthogonal. It can be imagined that the pixels of the image $\mathbf{x}_{2}$ are processed from top-left to bottom-right in $k$ steps where each step $\kappa$ is represented by the incremental transform $T_{\kappa}$. 


\section{A. The Incremental Transform}

Let $\mathbf{x}_{1}^{(\kappa)}$ and $\mathbf{x}_{2}^{(\kappa)}$ be two vectors representing consecutive pictures of an image sequence if $\kappa=1$, or two output vectors of the incremental transform $T_{\kappa-1}$ if $\kappa>1$. The incremental transform $T_{\kappa}$ maps these vectors according to

$$
\left(\begin{array}{l}
\mathbf{x}_{1}^{(\kappa+1)} \\
\mathbf{x}_{2}^{(\kappa+1)}
\end{array}\right)=T_{\kappa}\left(\begin{array}{l}
\mathbf{x}_{1}^{(\kappa)} \\
\mathbf{x}_{2}^{(\kappa)}
\end{array}\right)
$$

into two vectors $\mathbf{x}_{1}^{(\kappa+1)}$ and $\mathbf{x}_{2}^{(\kappa+1)}$ which will be further transformed into the temporal low- and high-band, respectively.

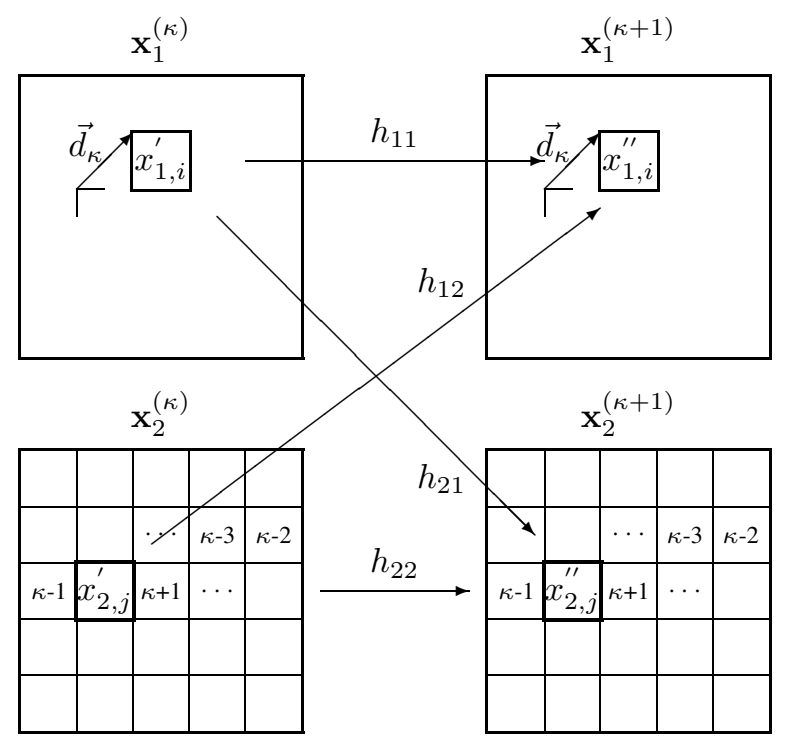

Fig. 1. The incremental transform $T_{\kappa}$ for two frames $\mathbf{x}_{1}^{(\kappa)}$ and $\mathbf{x}_{2}^{(\kappa)}$ which strictly maintains orthogonality for any motion field between the two frames.

Fig. 1 depicts the process accomplished by the incremental transform $T_{\kappa}$ with its input and output images as defined above. The incremental transform removes the energy of the $j$-th pixel $x_{2, j}^{\prime}$ in the image $\mathbf{x}_{2}^{(\kappa)}$ with the help of the $i$-th pixel $x_{1, i}^{\prime}$ in the image $\mathbf{x}_{1}^{(\kappa)}$ which is linked by the motion vector $\vec{d}_{\kappa}$ (or of the $j$-th block with the help of the $i$-th block if all the pixels of the block have the same motion vector $\left.\vec{d}_{\kappa}\right)$. The energy-removed pixel value $x_{2, j}^{\prime \prime}$ is obtained by a linear combination of the pixel values $x_{1, i}^{\prime}$ and $x_{2, j}^{\prime}$ with scalar weights $h_{21}$ and $h_{22}$. The energy-concentrated pixel value $x_{1, i}^{\prime \prime}$ is also obtained by a linear combination of the pixel values $x_{1, i}^{\prime}$ and $x_{2, j}^{\prime}$ but with scalar weights $h_{11}$ and $h_{12}$. All other pixels are simply kept untouched.

The scalar weights $h_{\mu \nu}$ are arranged into the matrix

$$
H=\left(\begin{array}{ll}
h_{11} & h_{12} \\
h_{21} & h_{22}
\end{array}\right)
$$

which is required to be orthogonal. For a $2 \times 2$ matrix, one scalar decorrelation factor $a_{n}$ is sufficient to capture all possible orthogonal transforms. We use the form

$$
H=\frac{1}{\sqrt{1+a_{n}^{2}}}\left(\begin{array}{cc}
1 & a_{n} \\
-a_{n} & 1
\end{array}\right)
$$

where $a_{n}$ is a positive real value to remove the energy in the image $\mathbf{x}_{2}$ and to concentrate the energy in the image $\mathbf{x}_{1}$. The decorrelation factor $a_{n}$ will be determined in the next subsection which discusses the energy-concentration constraint.

To summarize, the incremental transform $T_{\kappa}$ touches only pixels that have the same motion vector. Of these, $T_{\kappa}$ performs only a linear combination with pixels pairs that are connected by this motion vector. All other pixels are simply kept untouched. This is reflected with the following matrix notation

$$
T_{\kappa}=\left(\begin{array}{ccccccccc}
\ddots & \vdots & \vdots & \vdots & \vdots & \vdots & \vdots & \vdots & \vdots \\
\cdots & 1 & 0 & 0 & \cdots & 0 & 0 & 0 & \cdots \\
\cdots & 0 & h_{11} & 0 & \cdots & 0 & h_{12} & 0 & \cdots \\
\cdots & 0 & 0 & 1 & \cdots & 0 & 0 & 0 & \cdots \\
\vdots & \vdots & \vdots & \vdots & \ddots & \vdots & \vdots & \vdots & \vdots \\
\cdots & 0 & 0 & 0 & \cdots & 1 & 0 & 0 & \cdots \\
\cdots & 0 & h_{21} & 0 & \cdots & 0 & h_{22} & 0 & \cdots \\
\cdots & 0 & 0 & 0 & \cdots & 0 & 0 & 1 & \cdots \\
\vdots & \vdots & \vdots & \vdots & \vdots & \vdots & \vdots & \vdots & \ddots
\end{array}\right),
$$

where the diagonal elements equal to 1 represent the untouched pixels and where the elements $h_{\mu \nu}$ represent the pixels subject to linear operations.

Note that for accomplishing the transform $T$, each pixel in $\mathbf{x}_{2}$ is touched only once whereas the pixels in $\mathbf{x}_{1}$ may be touched multiple times or never. Further, the order in which the incremental transforms $T_{\kappa}$ are applied does not affect the orthogonality of $T$. But the order may affect the energy concentration of the transform $T$.

\section{B. The Constraint of Energy Concentration}

The decorrelation factor $a_{n}$ for each pixel touched by the incremental transform has to be chosen such that energy is removed from the image $\mathbf{x}_{2}$. We discuss a method that reduces the energy in the high-band to zero for any motion vector field if the input pictures are identical and of constant intensity.

Consider the pixel pair $x_{1, i}$ and $x_{2, j}$ to be processed by the incremental transform $T_{\kappa}$. To determine the decorrelation factor $a_{n}$ for the pixel $x_{2, j}$, we assume that the pixel $x_{2, j}$ is connected to the pixel $x_{1, i}$ such that $x_{2, j}=x_{1, i}$. Consequently, the resulting "to be high-band" pixel $x_{2, j}^{\prime \prime}$ shall be zero. Note that the pixel $x_{1, i}$ may have been processed previously by $T_{\tau}$, where $\tau<\kappa$. Therefore, let $v_{n}$ be the scale factor for the pixel $x_{1, i}$ such that $x_{1, i}^{\prime}=v_{n} x_{1, i}$. The pixel $x_{2, j}$ is used only once during the transform process $T$ and no scale factor needs to be considered, i.e., $x_{2, j}^{\prime}=x_{2, j}$. Let $v_{m}$ be the scale factor for the pixel $x_{1, i}$ after it has been processed by $T_{\kappa}$. Now, the pixels $x_{1, i}^{\prime}$ and $x_{2, j}^{\prime}$ are processed by $T_{\kappa}$ as follows:

$$
\left(\begin{array}{c}
v_{m} x_{1, i} \\
0
\end{array}\right)=\frac{1}{\sqrt{1+a_{n}^{2}}}\left(\begin{array}{cc}
1 & a_{n} \\
-a_{n} & 1
\end{array}\right)\left(\begin{array}{c}
v_{n} x_{1, i} \\
x_{1, i}
\end{array}\right)
$$

The condition of energy concentration is satisfied if

$$
\begin{aligned}
a_{n} & =\frac{1}{v_{n}} \text { and } \\
v_{m} & =\sqrt{v_{n}^{2}+1}
\end{aligned}
$$


Now, let $n$ be the scale counter. $n$ simply counts how often the pixel $x_{1, i}$ is used as reference for motion compensation. In the beginning, i.e., before the transform is applied, the scale counter for each pixel $x_{1, i}$ is $n=0$ and the scale value is $v_{0}=1$. With a scale counter $n$ for each pixel $x_{1, i},(9)$ and (8) simplify to $v_{n}=\sqrt{n+1}$ and $a_{n}=\frac{1}{\sqrt{n+1}}$, respectively.

The above mentioned condition of energy concentration is applicable only to pictures of the first level of the temporal decomposition. At the first level, each pixel $x_{2, j}$ of the picture $\mathbf{x}_{2}$ is used only once during the transform process. Therefore, there is no need to maintain a scale counter for the pixels in the picture $\mathbf{x}_{2}$. But at the second level of temporal decomposition, both pictures $\mathbf{x}_{1}$ and $\mathbf{x}_{2}$ are temporal low-bands resulting from transforms at the first level. Therefore, we need to consider scale factors $v_{n_{1}}$ and $v_{n_{2}}$ for the pixels $x_{1, i}$ and $x_{2, j}$ at higher levels of the temporal decomposition.

Let $v_{n_{1}}$ and $v_{n_{2}}$ be the scale factors for the pixels $x_{1, i}$ and $x_{2, j}$ that have been processed previously by $T_{\tau}$, where $\tau<\kappa$. Let $v_{m}$ be the scale factor for the pixel $x_{1, i}$ after it has been processed by $T_{\kappa}$. There is no need for an updated scale counter which corresponds to the pixels $x_{2, j}$ as they are touched only once during the transform process. To determine the decorrelation factors $a_{n}$ of a transform $T_{\kappa}$ at higher levels of the dyadic decomposition, we assume identical pictures of constant intensity as the input of the transforms at the first level. Again, we design a transform at a higher level of the dyadic decomposition such that the resulting "to be high-band" $\mathbf{x}_{2}^{(\kappa+1)}$ is zero. Now, the pixels $x_{1, i}$ at higher levels of the dyadic decomposition are processed by $T_{\kappa}$ as follows:

$$
\left(\begin{array}{c}
v_{m} x_{1, i} \\
0
\end{array}\right)=\frac{1}{\sqrt{1+a_{n}^{2}}}\left(\begin{array}{cc}
1 & a_{n} \\
-a_{n} & 1
\end{array}\right)\left(\begin{array}{c}
v_{n_{1}} x_{1, i} \\
v_{n_{2}} x_{1, i}
\end{array}\right)
$$

The condition of energy concentration is satisfied if

$$
\begin{aligned}
a_{n} & =\frac{v_{n_{2}}}{v_{n_{1}}} \text { and } \\
v_{m} & =\sqrt{v_{n_{1}}^{2}+v_{n_{2}}^{2}} .
\end{aligned}
$$

According to the definition of the scale counters at the first level of dyadic decomposition, the resulting scale factors are $v_{n_{1}}=\sqrt{n_{1}+1}$ and $v_{n_{2}}=\sqrt{n_{2}+1}$. For the second level, the condition of energy concentration in (12) requires the scale factors to satisfy $v_{m}=\sqrt{n_{1}+1+n_{2}+1}$. This result allows us to extend the definition of the scale counter to be applicable to any level of a dyadic decomposition if the scale counter $m$ of the next higher level of dyadic decomposition satisfies the following scale counter update rule:

$$
m=n_{1}+n_{2}+1
$$

Consequently, the scale factor at the second level is $v_{m}=$ $\sqrt{m+1}$ with the scale counter update rule. Moreover, the relation between the scale counter and the scale factor is valid for any level of a dyadic decomposition.

$$
v_{n}=\sqrt{n+1}
$$

This allows us to state the condition of energy concentration in terms of the scale counter. The decorrelation factor is

$$
a_{n}=\frac{\sqrt{n_{2}+1}}{\sqrt{n_{1}+1}}
$$

with the scale counters $n_{1}$ and $n_{2}$ which are maintained according to (13).

\section{Adaptive Spatial Transform}

The spatial transform that further decomposes the temporal low-band has to consider the scale factors that have been used during the temporal decomposition. In the following, we outline an adaptive spatial transform that is suitable.

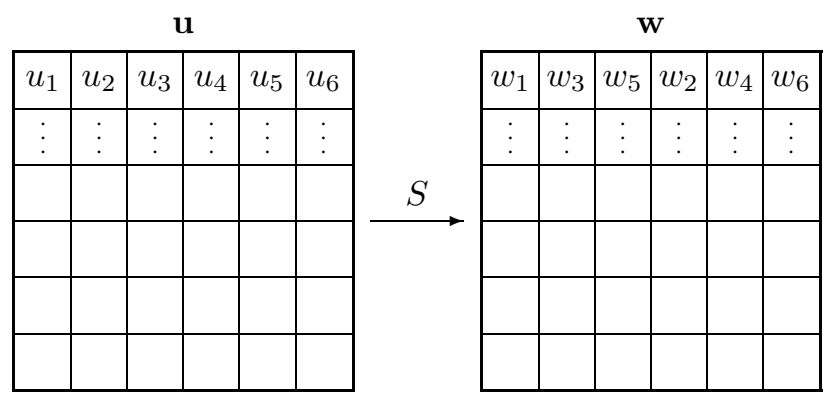

Fig. 2. The adaptive horizontal transform $S$ for a temporal low-band $\mathbf{u}$

Fig. 2 depicts the temporal low-band $\mathbf{u}$ and its horizontal decomposition $\mathbf{w}$. Let $u_{2 r+1}$ and $u_{2 r+2}$ be the odd and even horizontal samples of the the temporal low-band $\mathbf{u}$. The adaptive spatial transform $S$ maps these pixels according to

$$
\left(\begin{array}{l}
w_{2 r+1} \\
w_{2 r+2}
\end{array}\right)=S\left(\begin{array}{l}
u_{2 r+1} \\
u_{2 r+2}
\end{array}\right)
$$

into spatial low- and high-band coefficients $w_{2 r+1}$ and $w_{2 r+2}$, respectively. The transform matrix $S$ is the same as the matrix $H$ in (5). The decorrelation factor $a_{n}$ is also determined by (15), where $n_{2}$ denotes the scale counter of the even pixels and $n_{1}$ that of the odd pixels in the picture $\mathbf{u}$. The scale counter update rule for the spatial decomposition is also given by (13), where $m$ depicts the updated scale counter for the odd pixels in the picture $\mathbf{w}$.

After updating the scale counter, the scale counter for the even pixels in the picture $\mathrm{w}$ are set to zero. Consequently, a standard Haar transform $\left(a_{n}=1\right)$ is applied to obtain the horizontal and diagonal subbands. But an adaptive vertical transform is used to obtain the low-pass and the vertical subband. The adaptive vertical transform is analogous to the adaptive horizontal transform.

\section{EXPERIMENTAL RESUlts AND DisCUSSION}

Experimental results assessing the energy compaction are obtained for the QCIF sequences Foreman, Bus, and Mother \& Daughter. Our coding scheme with the motion-compensated orthogonal transform is compared to schemes which use a motion-compensated lifted Haar wavelet with and without update step. In addition, the performance of closed loop coding with hierarchical $\mathrm{P}$ pictures is reported. 


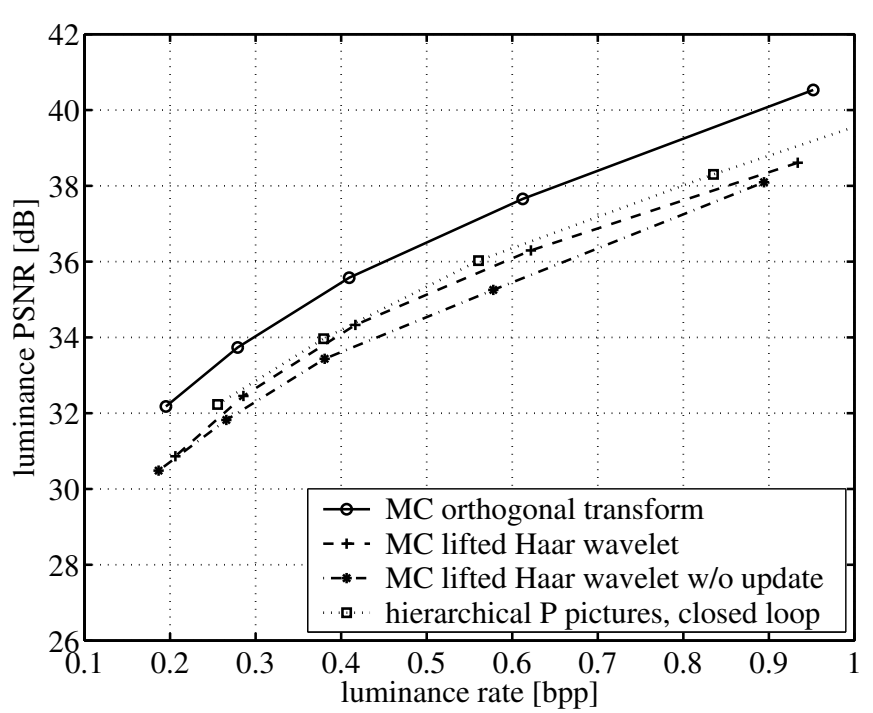

Fig. 3. PSNR over the rate for the luminance signal of the QCIF sequence Foreman at $30 \mathrm{fps}$ with 288 frames.

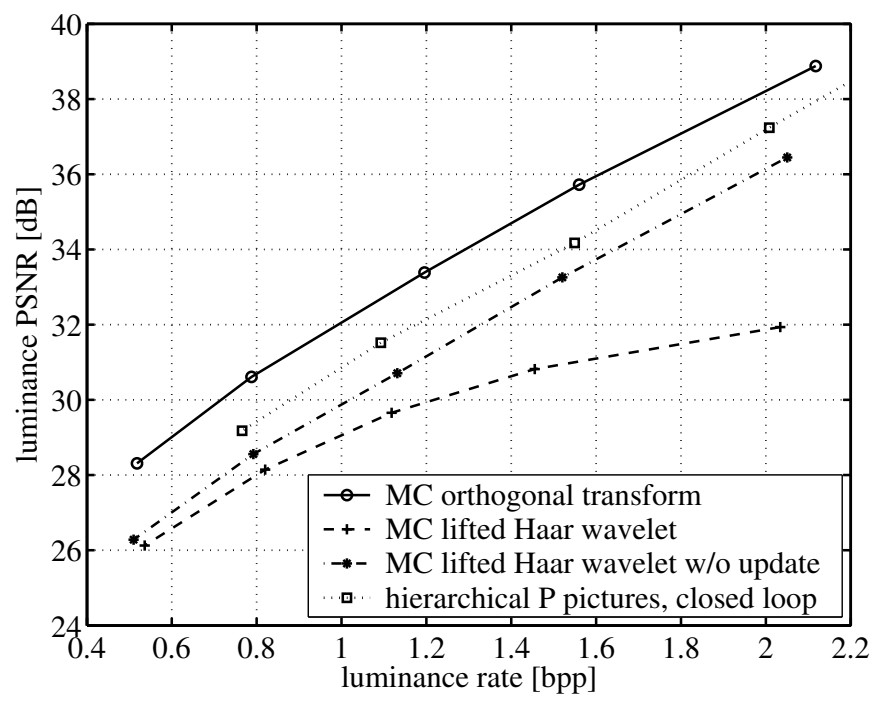

Fig. 4. PSNR over the rate for the luminance signal of the QCIF sequence Bus at 15 fps with 64 frames.

For the coding process with the orthogonal transform, a scale counter $n$ is maintained for every pixel of each picture. The scale counters are an immediate results of the utilized motion vectors and are only required for the processing at encoder and decoder. The scale counters do not have to be encoded as they can be recovered from the motion vectors.

All schemes operate with a GOP size of 16 frames. The block size for motion compensation is limited to $8 \times 8$. For simplicity, the resulting temporal subbands are coded with JPEG 2000. The temporal high-bands are coded directly, whereas the temporal low-band is re-scaled with (14) before encoding. For optimal rate allocation, Lagrangian costs are determined where the distortion term considers the scale factors applied to the temporal low-band.

Figs. 3, 4, and 5 depict the rate distortion performances for the luminance signals of the test sequences. Results for the

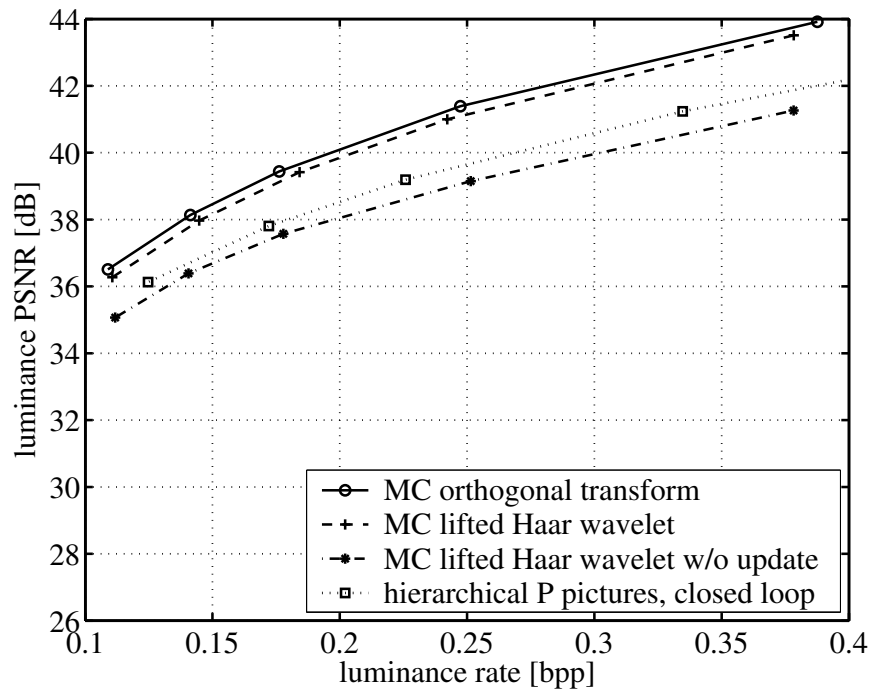

Fig. 5. PSNR over the rate for the luminance signal of the QCIF sequence Mother \& Daughter at $30 \mathrm{fps}$ with 64 frames.

orthogonal transform, the lifted Haar wavelet with and without update step, as well as for closed loop coding with hierarchical $\mathrm{P}$ pictures are given. For all test sequences, the orthogonal transform outperforms the reference schemes. For Bus, the significant motion in the sequence degrades the performance of the lifted Haar wavelet as the update step introduces substantial noise. For Mother \& Daughter, the orthogonal transform outperforms the lifted Haar wavelet with update step by a small margin as the weak motion in the sequence does not substantially harm the lifted Haar wavelet.

\section{COnClusions}

This paper presents a motion-compensated orthogonal transform which strictly maintains orthogonality for any motion field. It outperforms the motion-compensated lifted Haar wavelet which is not able to maintain orthogonality in all cases. The orthogonality principle improves energy compaction and provides a highly robust video representation.

\section{ACKNOWLEDGEMENT}

The authors thank Aditya Mavlankar for helpful discussions.

\section{REFERENCES}

[1] J.-R. Ohm, "Three-dimensional subband coding with motion compensation," IEEE Transactions on Image Processing, vol. 3, no. 5, pp. 559-571, Sept. 1994.

[2] S.-J. Choi and J. Woods, "Motion-compensated 3-d subband coding of video," IEEE Transactions on Image Processing, vol. 8, no. 2, pp. 155167, Feb. 1999.

[3] B. Pesquet-Popescu and V. Bottreau, "Three-dimensional lifting schemes for motion compensated video compression," in Proceedings of the IEEE International Conference on Acoustics, Speech and Signal Processing, vol. 3, Salt Lake City, UT, May 2001, pp. 1793-1796.

[4] A. Secker and D. Taubman, "Motion-compensated highly scalable video compression using an adaptive 3D wavelet transform based on lifting," in Proceedings of the IEEE International Conference on Image Processing, vol. 2, Thessaloniki, Greece, Oct. 2001, pp. 1029-1032.

[5] M. Flierl and B. Girod, "Video coding with motion-compensated lifted wavelet transforms," Signal Processing: Image Communication, vol. 19, no. 7, pp. 561-575, Aug. 2004.

[6] B. Girod and S. Han, "Optimum update for motion-compensated lifting,' IEEE Signal Processing Letters, vol. 12, no. 2, pp. 150-153, Feb. 2005. 